\title{
Software Architecture of Code Analysis Frameworks Matters: The Frama-C Example
}

\author{
Julien Signoles* \\ CEA LIST, Software Security Lab, \\ PC 174, 91191 Gif-sur-Yvette, France \\ julien.signoles@cea.fr
}

\begin{abstract}
Implementing large software, as software analyzers which aim to be used in industrial settings, requires a well-engineered software architecture in order to ease its daily development and its maintenance process during its lifecycle. If the analyzer is not only a single tool, but an open extensible collaborative framework in which external developers may develop plug-ins collaborating with each other, such a well designed architecture even becomes more important.

In this experience report, we explain difficulties of developing and maintaining open extensible collaborative analysis frameworks, through the example of Frama- $C$, a platform dedicated to the analysis of code written in $\mathrm{C}$. We also present the new upcoming software architecture of Frama-C and how it aims to solve some of these issues.
\end{abstract}

\section{Introduction}

This experience report is about software architecture. Software architecture may be defined as the set of structures needed to reason about the system which comprise software elements, relations among them, and properties of both [1]. It has long been recognized that implementing a large piece of software without a well-engineered software architecture will stumble along or, most likely, fail [2]. Thus, whatever the software is, its architecture does matter. This article aims at providing a practical evidence about this statement through a concrete example.

This short paper is not only about software architecture. It is also about code analysis tools and formal methods based tools. Code analysis is known to be complex and difficult. In particular when based on formal methods. Besides formal methods are still a very active research domain in which new techniques and improvements are discovered frequently. Consequently tools based on such techniques evolve quickly, or become outdated quickly. Despite this harsh reality, some analysis tools become mature enough to be used in industrial settings. Such tools must combine the intrinsic hardness of code analysis with the intricacies of industrial code they aim to analyze. Thus they are large and complex pieces of software. So they need well-engineered software architectures which must permit tools to fulfill two opposite requirements: be flexible enough to allow rapid prototyping and experimentation; be stable enough to obtain reproducible predictable results and no regression after upgrading.

Few code analysis tools are code analysis frameworks. Frameworks can be characterized by few key features. First extensibility which allows to extend the framework with new features. Then collaboration which allows to mix extensions to provide new super features quickly and easily. Next inversion

${ }^{*}$ This work has been partly supported by the European FP7 project Stance 317753.

C. Dubois, P. Masci, D. Méry (Eds): 2nd International Workshop on Formal Integrated Development Environment (F-IDE 2015)

EPTCS 187, 2015, pp. 86-96 doi 10.4204/EPTCS.187.7 (c) J. Signoles

This work is licensed under the Creative Commons Attribution License. 
of control which gives the control of the overall application to the framework itself and not to the application that uses it [12]. Finally opening, usually via open-sourcing, which allows external developers to contribute.

Perhaps not surprisingly, developing and maintaining a large flexible yet mature open extensible collaborative code analysis framework is not that easy. But thankfully a software architecture may be very helpful if carefully designed. The goal of this short paper is to provide evidence about these statements, through the example of Frama-C [13], which is such a framework dedicated to the analysis of code written in $C$.

Section 2 quickly introduces Frama-C. Section 3 details requirements which must be fulfilled to provide the framework key features. Section 4 presents the evolutions of the Frama-C architectures up to the upcoming one and explains which requirements they try to address.

\section{Frama-C at a Glance}

Frama- $C$ [13] is a platform dedicated to the analysis of source code written in $C$. The Frama- $C$ platform gathers several analysis techniques into a single collaborative extensible framework. For instance it contains Value, which is a value analysis by abstract interpretation [8], WP, which is a program proof tool through weakest precondition calculus [4], and E-ACSL which is a monitoring tool [9].

The analyzers share a common specification language called ACSL [3]: any analyzer can prove the (un)validity of each ACSL annotation of a $C$ program, but they can also generate them. That is one way to ensure analyzer collaboration. For instance the RTE tool [11] is able to generate an annotation for each potential runtime error, and then Value, WP or E-ACSL can try to prove each of them, while Frama$C$ itself combines what is proven by any single tool to compute what still remains to be proven on the whole [5]. Another way to allow analyzer collaboration is through the use of their OCaml programmatic interfaces ( $a k a$ APIs) by each other. For instance, the callgraph may use the results of Value to resolve function pointers.

Frama-C is also extensible: it is possible to develop new analyzers in OCaml and to integrate them in the framework. Anyone can do this thanks to its licensing policy (LGPL, version 2): Frama-C is open source and most of its plug-ins as well (for instance all of the above-mentioned plug-ins are open source), while it is still possible to implement close-source proprietary extensions [10, 6]. This way, Frama-C is not only a collection of collaborative tools but also a development platform in OCaml which targets both academic and industrial users and developers [7].

\section{Frama-C Requirements}

The Frama- $C$ overview induces nice properties that the tool must have. First, Frama- $C$ must be usable both from the command line and in a graphical user interface (GUI). Second it must analyze (ISO 99) $C$ code annotated with ACSL specifications. Consequently it must provide a $\boldsymbol{C}$ front-end, extended by an ACSL implementation. Particularly it must provide at least one abstract syntax tree (AST) from annotated $C$ files. Related to these requirements, Frama- $C$ must be easily identified as a single tool from a user point of view. That means primarily homogeneity. For instance, user interactions like getting inputs or printing messages should be as uniform as possible in order to make the learning of the tool easier and faster, while it must be possible to integrate new analyzers in the GUI smoothly. However Frama-C is also a collection of possibly very different tools: using a monitoring tool based on program transformation techniques is not the same as using a program proof tool and both are different 
from an abstract interpreter in many aspects. This heterogeneity of uses means that Frama-C must be customizable enough to fit the analyzer needs. Being both homogeneous and heterogeneous while being user friendly implies that the user should identify quickly what is common to all analyzers and what is specific to each of them. For instance, a code analysis tool has usually many parameters which allow the user to tweak the tool according to its current use case. In our context, it must be able to quickly know which parameters are common to all analyzers (e.g. the hypotheses used to compute system-dependent information like the size of $C$ types) and which ones are relevant only for a specific (set of) analyzer(s) (e.g. the slicing criteria used by the slicing tool).

Analyzer collaboration is provided by two means. The first one is exchanging ACSL annotations: analyzers must be able to generate new annotations when required (for instance to make explicit their correctness hypotheses) which several other analyzers could try to verify. In this context, the kernel ensures global consistency by consolidating which property is proved by which analyzer under which hypotheses [5]. The second way of analyzer collaboration is through APIs: a plug-in may directly use values (including functions) exported by others plug-ins either to tweak their behaviors or to compose them to quickly develop powerful analyzers.

In order to ensure homogeneity and inversion of control, Frama- $C$ itself must control the overall execution of the tool and not let each individual analyzer decide. For instance, Frama- $C$ must parse itself the command line, display messages, run the GUI, parse the source code, run the analyzers, etc. However it must let place to analyzer customization when it makes sense.

Furthermore Frama- $C$ must be not only a tool but also a large library which must provide useful services to ease analyzer developments. It must be developed in OCaml [7].

\section{Frama-C Architectures}

While the first Frama-C public release was in 2008, Frama- $C$ is developed since 2005 and 3 different architectures have been successively designed. The prehistoric one, presented in Section 4.1, was used from the beginning of the development until 2007. It was then replaced by the current architecture, detailed in Section 4.2. It is now in turn going to be replaced by the upcoming one explained in Section 4.3 . Section 4.4 estimates the cost of these evolutions.

\subsection{Prehistoric Architecture}

The Frama- $C$ architecture is from the origin based on a single fundamental principle: it is a plug-in oriented architecture à la Eclipse ${ }^{1}$ in which each analyzer is a plug-in which uses a kernel. This design choice is nowadays the standard way to provide extensibility while helping to solve the homogeneity $v s$ heterogeneity issue: the kernel ensures some homogeneity while plug-ins may develop their own concepts when required. Analyzer collaboration through API is ensured by the kernel which maintains a plug-in database storing the API functions. However, at the very beginning of the Frama-C development, this principle was more an essential principle than a perceptible reality expressed by Figure 1 .

Indeed plug-in developers have to modify themselves some parts of the kernel (particularly the plugin database and the Makefile) to register new analyzers, while it was not possible to plug or unplug them without recompiling the whole tool. More generally, there were no clear distinction between plug-ins and the kernel. There were at least two major reasons for such issues. First Frama- $C$ was initially developed in a hurry by a couple of persons to demonstrate that it could be a viable project which it is

\footnotetext{
http://eclipse.org/
} 


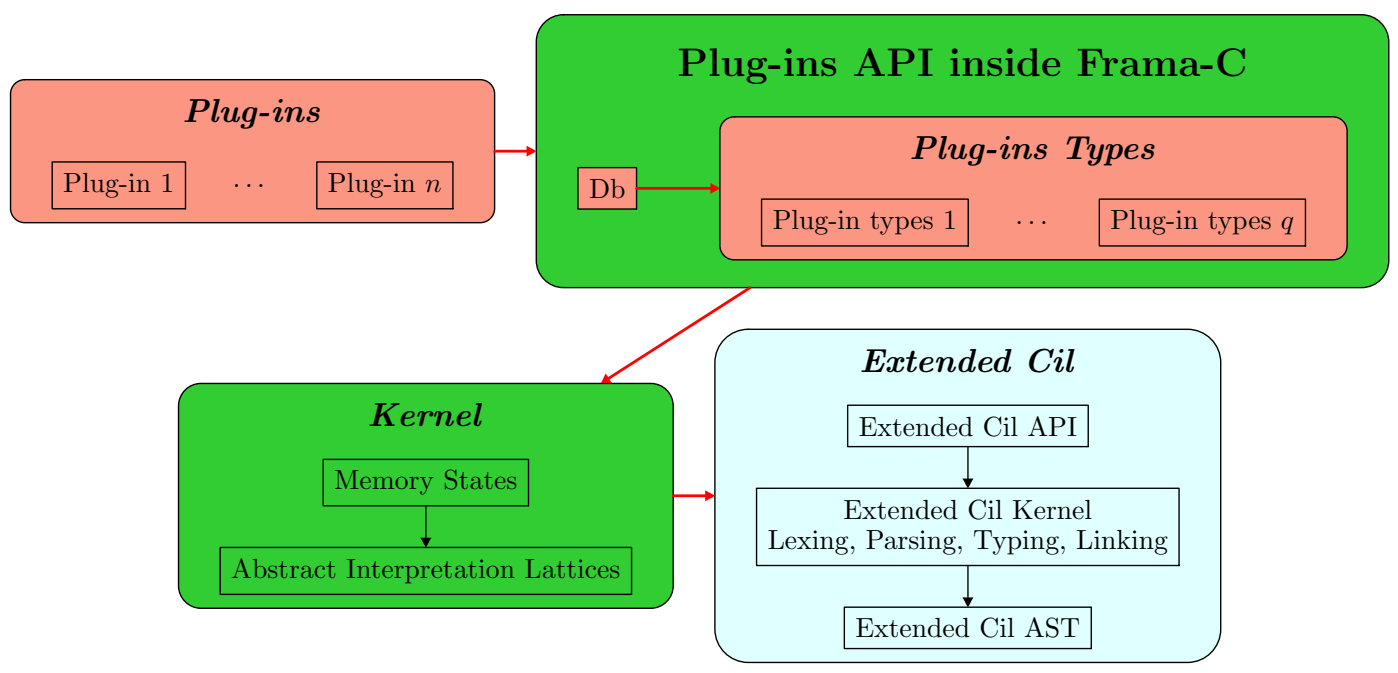

Figure 1: Frama-C Prehistoric Architectural Description

worth being founded. Second OCaml had no native dynamic linking at that time and thus a "plug-in" has to be statically linked against the rest of the platform. Also OCaml does not support mutually dependent compilation units: that complicates a bit the overall organization.

This plug-in oriented architecture naturally leads to inversion of control: the kernel controls the Frama- $C$ execution and decides by its own when each part of each plug-in must be executed. For instance, one part of a plug-in is ran when parsing the command line to handle plug-in specific options and another part is ran to execute the main function of the analyzer.

The other main design choice of the prehistoric architecture is the use of $C I L$ [14] as $C$ front-end. $C I L$ is also both a tool and a library but Frama- $C$ only uses the library part. It provides a $C$ AST from $C$ source files and an API to use it easily. This API is almost organized in a centralized way: a single large compilation unit ( $\mathrm{Cil}$ ) contains almost the whole API. At that time, Frama-C directly reuses $C I L$ as such, but extended it in order to support $A C S L$. Even if Frama-C integrated its own version of $C I L$, it was regularly synchronized with the Berkeley mainstream version. Finally, since the very first important plug-in was Value, the main library provided by the kernel was an abstract interpretation toolkit.

\subsection{Current Architecture}

Figure 2 introduces the Frama-C architectural description which was defined in 2007. It is still in use in the last open source release of March 2015 (namely Sodium). When defined, it was explained in a document which more generally explains how to develop Frama- $C$ plug-ins [17].

Thanks to the introduction of native dynamic loading into OCaml, it becomes possible to provide fully independent plug-ins developed outside the platform without any modification of the kernel nor recompilation, even if the old ways of developing plug-ins is still supported. However OCaml is a statically typed programming language. That leads to technical issues with APIs, packing facilities ${ }^{2}$ and dynamic loading which limit plug-in collaborations through API to simple uses. In particular, registering

\footnotetext{
${ }^{2} \mathrm{An} \mathrm{OCaml} \mathrm{pack} \mathrm{is} \mathrm{a} \mathrm{way} \mathrm{to} \mathrm{group} \mathrm{together} \mathrm{at} \mathrm{compile} \mathrm{time} \mathrm{a} \mathrm{set} \mathrm{of} \mathrm{compilation} \mathrm{units} \mathrm{into} \mathrm{a} \mathrm{single} \mathrm{one.} \mathrm{In} \mathrm{Frama-C,} \mathrm{each}$ plug-in is compiled this way in order to not pollute the whole namespace with the names of the plug-in internal compilation units.
} 


\section{Plug-ins}
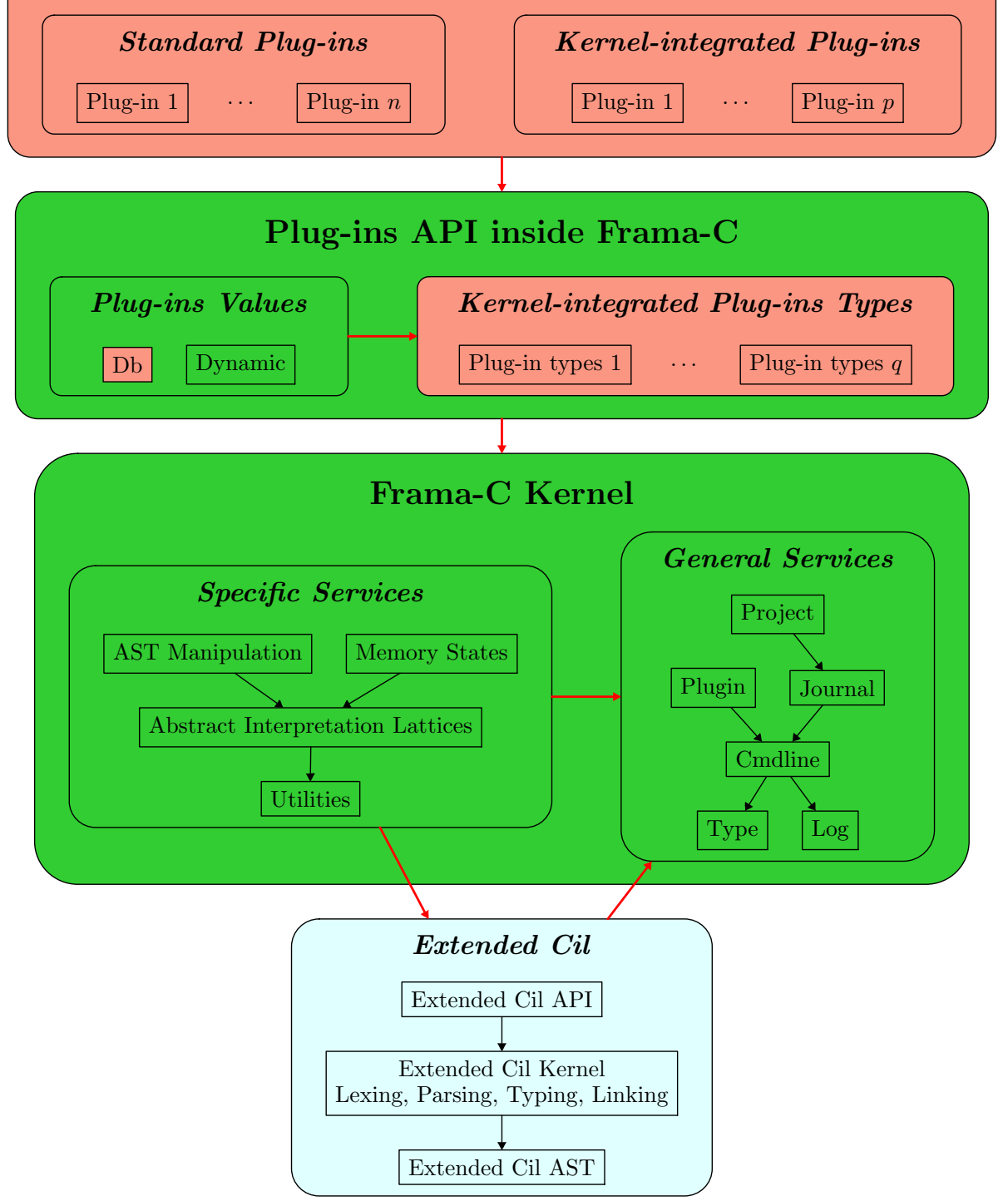

Figure 2: Current Architectural Description 
new types is limited to abstract types, while registering values is restricted to monomorphic values and remains tedious through the use of a dedicated library [16]. In practice, this limitation is tractable as long as no plug-in needs to export a large set of functions [7].

Within this architecture, the kernel is still based on CIL but it also provides its own services as libraries in order to simplify plug-in development and to ensure plug-in consistency. Just after the introduction of this architecture, CIL itself had been fully forked from Berkeley's mainstream: in addition to the support of ACSL, the changes introduced to be consistent with the other parts of the kernel (unified ways of displaying messages and handling errors, integration of the project system [15], etc) were so huge that synchronization with the Berkeley's version became intractable.

Inversion of control is an important feature of a framework, but a plug-in often needs to customize the default behavior. Such an a posteriori modification is not so easy to provide in a statically typed functional language like $O C a m l$. It is usually done by hooking which consists in registering a closure to be executed at a well defined moment of the execution. Yet these hooks do not appear as such in the architectural description. They have nevertheless a major impact in the overall organization of the framework since they get back some control to plug-ins at many places.

This architecture fulfills most requirements from plug-in collaborations to extensibility through heterogeneity of uses via customizability and global homogeneity of the platform via inversion of control. It probably contributes to the adoption of Frama- $C$ throughout the world. Frama- $C$ and its community - including plug-in developers - are still growing: the number of line of codes $(l o c)$ of the open source version as well as the number of plug-ins and developers (including third-party ones) constantly increase.

However that has revealed an important issue of the platform: non expert plug-in developers are often not able to find what they are looking for in the Frama-C API, and even do not know where to search in. Even expert developers working on Frama- $C$ since a long time face such difficulties regularly. This annoying situation is partly inherent to 100+-kloc libraries: searching one piece of information in a large code base is never easy, particularly when unknown. However, in our case, the quality of the kernel APIs also regularly deteriorate because it becomes more and more unclear where to add a new function or a new library. For instance, basic functions to manipulate the AST are dispatched in several files of different directories without any apparent logic.

Indeed huge libraries are usually organized in a decentralized way: they are split into lots of files, small enough to remain tractable. Frama- $C$ has been no exception to the rule while growing. However this organization clashes with the CIL centralized organization. Thus a large part of the kernel has no clear organization at all and no one is able to explain where is the best place to add. For instance, should a new function about some ACSL construct be added in CIL among plenty of other functions, or in a CIL extension, or in some other kernel files dedicated to ACSL? When it is unclear where to add a function, it is a fortiori unclear where to search for a value. Furthermore some (parts of) modules only address very specific needs or even should only be used by the kernel and does not target the standard plug-in developer. In particular, there are plenty of forward references in the kernel just to circumvent the absence of mutually recursive compilation units in OCaml. However they remain visible by anyone. These drawbacks contribute to the current issue.

Another issue is that Frama- $C$ is still in heavy development because it tries to remain at (or to reach) the cutting edge of scientific research: that leads to unavoidable instability of several parts of the framework. Of course it is annoying for any developer which has to maintain its plug-ins, even if we provide as far as possible automatic migration scripts.

Finally the last issue is that few plug-in developers begin wanting to provide a large API with tens of functions and types for their plug-ins and it is almost intractable with the current limitation of dynamic loading. For instance, one use case is to develop a plug-in whose the main goal is to be a dedicated 
library for other plug-ins.

\subsection{Upcoming Architecture}

To synthesize the previous section, the current architecture almost fulfills our needs, but we hugely need to help the developer to find what is looking for by providing a way to quickly and precisely know what every module and single function is useful for. Namespace is a standard way to address this issue at the programming language level. However OCaml has a poor support of namespaces even if there are currently intensive discussions around this topic in the OCaml community: for instance it is not possible to indicate that a value or a module can only be used by a single family of values or modules (like C++'s friendship or Java package visibility for instance). Therefore we decide to address this issue at the architecture level. This choice leads to the architecture presented Figure 3 which is currently deployed in a Git branch of the private development version of Frama-C. It is planned to be part of the next public release.

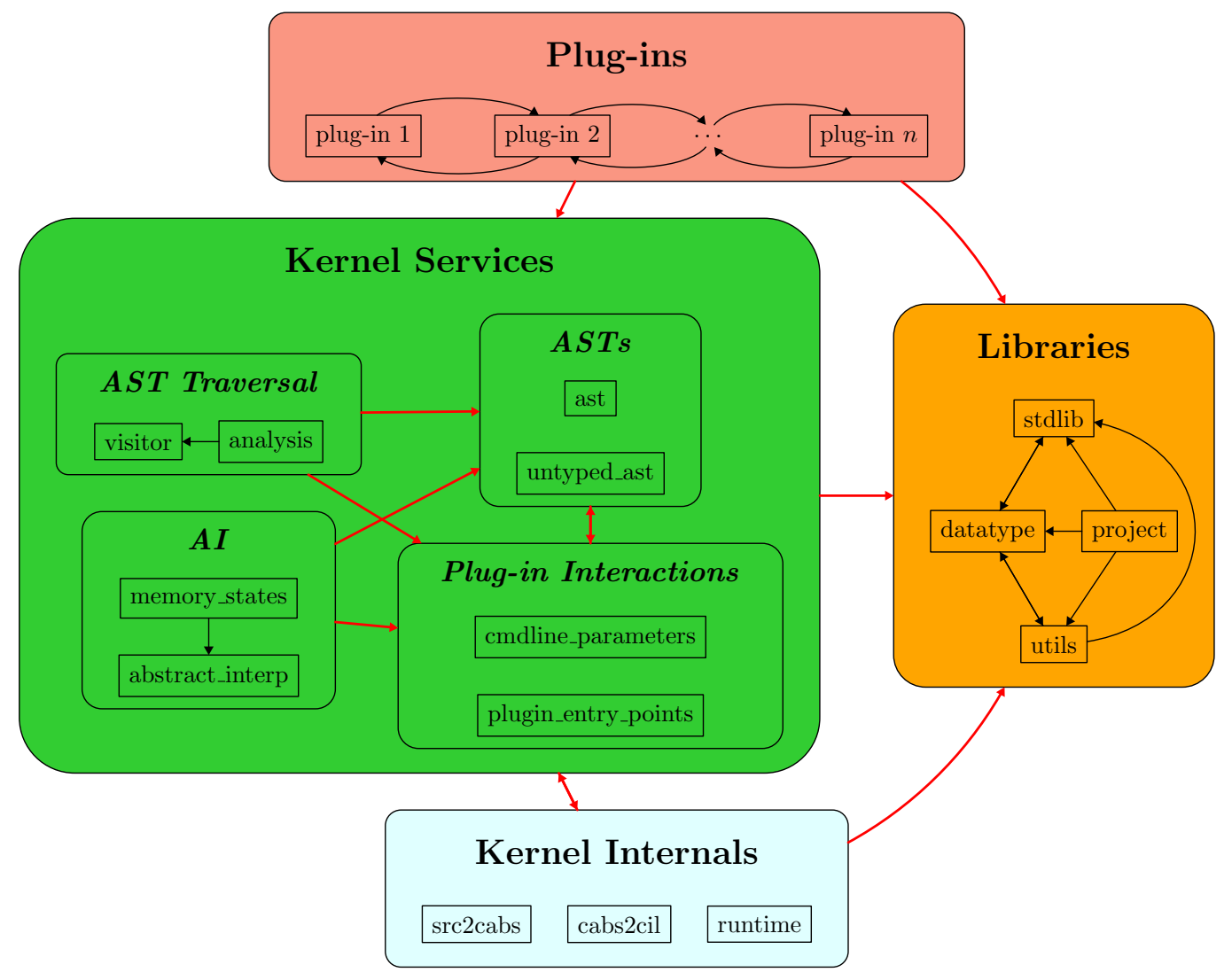

Figure 3: Upcoming Architectural Description

The main design idea of this new architecture is to split Frama- $C$ into distinct areas, clearly separated thanks to the directory structures. First plug-in (sub-)directories delivered with Frama- $C$ are no longer in the same directory than most kernel directories, but are group together in another directory. So there is a clear boundary between the kernel and the plug-ins which is visible at a glance by any developer. Second the kernel itself is split into three main areas: Libraries, Kernel Services and Kernel Internals. Libraries 
contains modules which are not specific to code analysis and could be moved outside Frama- $C$ easily (e.g. extension of the $O C \mathrm{Caml}$ standard libraries, general datastructures like bags or bit vectors, wrapping of system commands, etc). Kernel Services provides useful services to plug-in developers (e.g. access to the AST, abstract interpretation toolkit, standard interaction points between plug-ins and the kernel, etc). Kernel Internals contains modules which should be useless for plug-in developers (except for very advanced uses reserved to experts through hooking) like the generation of the ASTs from a $C$ source code or the routines run when initializing Frama- $C$. This design idea should almost solve the previously identified issues by reducing and easily identifying the place to look for when searching something in the API. For instance, no need to search in the kernel internals anymore, while a developer may not even easily know that a module should not be used outside the kernel in the previous architecture.

A major consequence of this new architecture is to remove CIL: even if the implementation is still the same, it does not appear anymore in the whole architectural description. Yet deploying the new architecture is a two-step stage and only the first one is being finalized. It only modifies the directory level, without changing the module APIs. Thus it has no impact on the existing plug-ins based on the Frama-C API. The second step is still not precisely planned in our timeline but it will remove the $C I L$ centralized organization by modifying Frama-C API. In order to ease the migration from old versions to the newest one, we will design new services one at a time from existing functionalities which are currently dispatched at several places of CIL. Then CIL will be removed when it will be rendered meaningless and small enough. Past experiences with the (re)design of other Frama- $C$ services have already shown that it is often the less painful way to introduce major incompatibilities between releases. It will eventually solve the $C I L$-centralized $v s$ kernel-decentralized issue. It could be pointed out that it could deserve the adoption of the platform by existing CIL users, but mainstream CIL and the forked CIL version of Frama- $C$ have diverged enough to already be an issue with the current architecture. Also the benefits for all the Frama- $C$ developers community should be much larger than this potential drawback for a small part of the community.

Finally the last action to help developers to find out about what they are looking for is to improve what we could call the micro software architecture: API organization in a single compilation unit. As already explained, such a unit often contains hooks, parts dedicated to kernel or advanced uses, (almost) stable parts or parts which could still evolve in the near future, etc. At this level, we are continuously updating the API to clearly separate and document each part in order to indicate the functionalities from the more idiomatic ones to the niches one. For instance, we put at the very end of the module API the kernel-only features and they are even hidden in the generated HTML documentation.

The other evolution which comes from this new architecture is the interactions between plug-ins. The limitations of plug-in APIs almost disappeared thanks to OCaml's first class modules 3 This OCaml evolution allows Frama- $C$ to replace the previous heavy interaction mechanism through a standard communication via file interfaces. There is still room for improvements here, but the previous mechanisms are nowadays almost deprecated, and their uses will be gradually replaced. It will eventually allow plug-ins to provide an API as large as they wish and thus to solve the last issue mentioned in Section 4.2

\subsection{Estimation Cost of the Changes}

Estimating the manpower required to deploy the current and the upcoming architecture is not that easy mainly because it depends on what is counted and what is not counted. Here I do not take into account

\footnotetext{
${ }^{3}$ They were first included in OCaml 3.12.0 released on January 2012, but major evolutions in recent versions of OCaml makes them much easier to use.
} 
the development of new services like dynamic loading or the project system, but I do take into account file modifications (e.g. changes in Makefiles) required by the architectural changes.

The current architecture was implementing in 2007 by almost a single developer (the author of this article) in few weeks over several months. Most efforts was spent in clearly separating the plug-ins from the kernel in Makefiles and configures' scripts. Modifying the directories and files structure was not a big deal because Frama- $C$ was still in its early days at that time: there were not that many plug-ins nor developers.

Implementing the upcoming architecture is a more important effort which is still not ended. We have already spent several men-Weeks of developments and intensive discussions over 5 months just to define the architecture directory-level without talking so much about the file-level and the process is only closed to be finished in its first version. This large amount of time comes from there are more core developers than before and they may have divergent opinions. More importantly, the development is done in a separated Git branch along several months since no developer have enough time to only work on this topic. This branch only consists in creating and deleting directories and moving files. As expected, it has no impact on API and so on plug-in developers. However it may conflict a lot with other branches which do the same kind of operations since Git is really bad to merge two branches if both moves the same file in different ways (or if one branch moves the file and the other one deletes it). Unfortunately these patterns already occur several times (for instance, one merged branch reorganized non-regression test directories of a major plug-in). There were also heavy changes in the Makefile which conflicted with the Makefile changes of the branch of the new architecture. At the end, heavy Git conflicts happens much more frequently than expected.

The second stage of the new architecture deployment, which will modify the file APIs, is still not precisely planned in our timeline but it will be done part by part along months or even years of sparse developments on that topic.

\section{Conclusion}

Through the evolution of the Frama- $C$ architecture, this article has explained why software architecture matters. In particular, it has shown how software architecture may help to fulfill requirements of a code analysis frameworks and thus have a major impact in the daily life of its developers, including third party ones. Software architecture is only an element over several other engineering and technical choices. For instance, documentation is also crucial. Also software architecture is not only a black board exercise. It has a concrete representation which must take into account technical issues like the choice of the underlying programming language. Finally, the software architecture must evolve along with the whole software lifecycle and potentially be replaced when becoming outdated. However such a change has an huge impact on the whole software and must be made with care in order to limit annoyance. In this respect, change management techniques are useful to help the developers to migrate their code as easily as possible without mumbling too much, to understand the new architecture and eventually to accept such a major change.

An open plug-in based architecture is the key of the adoption of a tool like Frama- $C$ by both the academic and the industrial formal methods communities. However it is not so easy to deploy in practice and the sooner the better. Also, while reusing existing libraries saves a lot of time, they must be redesigned to be well integrated into the architecture as soon as it has to be extensively modified (like CIL in Frama-C). 


\section{Acknowledgments}

Special thanks to Virgile Prevosto and Boris Yakobowski who spend a large amount of their time to review the huge Git branch implementing the new architecture. I would also like to thank François Bobot and Loïc Correnson for their constructive feedback and suggestions about it as well as Florent Kirchner who provides a continued support to this major change. Furthermore the anonymous referees contribute to improve the final version of this paper thanks to their fruitful remarks.

\section{References}

[1] Felix Bachmann, Len Bass, Paul Clements, David Garlan, James Ivers, M. Little, Paulo Merson, Robert Nord \& Judith Stafford (2010): Documenting Software Architectures: Views and Beyond, second edition. AddisonWesley Professional. http://resources. sei.cmu.edu/library/asset-view. cfm?assetid=30386.

[2] Len Bass, Paul Clements \& Rick Kazman (2012): Software Architecture in Practice (3d Edition). AddisonWesley Professional. http://resources. sei. cmu.edu/library/asset-view.cfm?assetid=30264.

[3] Patrick Baudin, Jean-Christophe Filliâtre, Claude Marché, Benjamin Monate, Yannick Moy \& Virgile Prevosto (2015): ACSL: ANSI/ISO C Specification Language. Version 1.9. http://frama-c.com/download/ acsl_1.9.pdf

[4] Loc Correnson, Zaynah Dargaye \& Anne Pacalet (2015): Frama-C's WP plug-in. http://frama-c.com/download/frama-c-wp-manual.pdf

[5] Loc Correnson \& Julien Signoles (2012): Combining Analysis for C Program Verification. In: Formal Methods for Industrial Critical Systems (FMICS), doi:10.1007/978-3-642-32469-7_8.

[6] Pascal Cuoq, David Delmas, Stéphane Duprat \& Victoria Moya Lamiel (2012): Fan-C, a Frama-C plug-in for data flow verification. In: Embedded Real Time Software and Systems (ERTSS), http://web1.see. asso.fr/erts2012/Site/0P2RUC89/5C-3.pdf

[7] Pascal Cuoq \& Julien Signoles (2009): Experience Report: OCaml for an industrial-strength static analysis framework. In: Proceedings of International Conference of Functional Programming (ICFP'09), ACM Press, New York, NY, USA, pp. 281-286, doi $10.1145 / 1631687.1596591$

[8] Pascal Cuoq, Boris Yakobowski \& Virgile Prevosto (2015): Frama-C's value analysis plug-in. http://frama-c.cea.fr/download/value-analysis.pdf.

[9] Mickaël Delahaye, Nikolai Kosmatov \& Julien Signoles (2013): Common Specification Language for Static and Dynamic Analysis of C Programs. In: Symposium on Applied Computing (SAC'13), ACM, pp. 1230 1235, doi: $10.1145 / 2480362.2480593$

[10] David Delmas, Stéphane Duprat, Victoria Moya Lamiel \& Julien Signoles (2010): Taster, a Frama-C plug-in to encode Coding Standards. In: Embedded Real Time Software and Systems (ERTSS). http://web1.see.asso.fr/erts2010/Site/OANDGY78/Fichier/PAPIERS\%20ERTS\% 202010/ERTS2010_0003_final.pdf.

[11] Philippe Herrmann \& Julien Signoles (2013): Annotation Generation: Frama-C's RTE plug-in. http://frama-c.com/download/frama-c-rte-manual.pdf

[12] Ralph E. Johnson \& Brian Foote (1988): Designing Reusable Classes. Journal of Object-Oriented Programming 1(2), pp. 22-35. http://www.cse.msu.edu/ cse870/Input/SS2002/MiniProject/Sources/ DRC.pdf

[13] Florent Kirchner, Nikolai Kosmatov, Virgile Prevosto, Julien Signoles \& Boris Yakobowski (2015): FramaC: A Software Analysis Perspective. Formal Aspects of Computing, pp. 1-37, doi $10.1007 / \mathrm{s} 00165-014-0326-$ 7. 
[14] George C. Necula, Scott McPeak, Shree Prakash Rahul \& Westley Weimer (2002): CIL: Intermediate Language and Tools for Analysis and Transformation of C Programs. In: CC '02: Proceedings of the 11th International Conference on Compiler Construction, Springer-Verlag, London, UK, pp. 213-228, doi:10.1007/3540-45937-5_16

[15] Julien Signoles (2009): Foncteurs impératifs et composés: la notion de projet dans Frama-C. In Hermann, editor: Journées Francophones des Langages Applicatifs, Studia Informatica Universalis 7.2, pp. 245-280. In French. http://www-ist.cea.fr/publicea/exl-doc/200800005272.pdf

[16] Julien Signoles (2011): Une bibliothèque de typage dynamique en OCaml. In Hermann, editor: Journées Francophones des Langages Applicatifs, Studia Informatica Universalis, pp. 209-242. In French. http: //studia.complexica.net/Art/AC-JFLA11-08.pdf.

[17] Julien Signoles, Loc Correnson, Matthieu Lemerre \& Virgile Prevosto (2015): Frama-C Plug-in Development Guide.

http://frama-c.cea.fr/download/plugin-developer.pdf 